\title{
Magnetic field distribution and characteristic fields of the vortex lattice for a clean superconducting niobium sample in an external field applied along a three-fold axis
}

\author{
A. Yaouanc, ${ }^{1}$ A. Maisuradze,${ }^{2,3}$ N. Nakai, ${ }^{4}$ K. Machida, ${ }^{4}$ R. Khasanov,${ }^{3}$ A. Amato, ${ }^{3}$ P. K. Biswas, ${ }^{3}$ C. Baines,${ }^{3}$ \\ D. Herlach, ${ }^{3}$ R. Henes, ${ }^{5}$ P. Keppler, ${ }^{5}$ and H. Keller ${ }^{2}$ \\ ${ }^{1}$ Institut Nanosciences et Cryogénie, SPSMS, CEA and Université Joseph Fourier, F-38054 Grenoble, France \\ ${ }^{2}$ Physik-Institut der Universität Zürich, Winterthuerstrasse 190, CH-8057 Zürich, Switzerland \\ ${ }^{3}$ Laboratory for Muon-Spin Spectroscopy, Paul Scherrer Institute, CH-5232 Villigen-PSI, Switzerland \\ ${ }^{4}$ Department of Physics, Okayama University, Okayama 700-8530, Japan \\ ${ }^{5}$ Max-Planck-Institut für Metallforschung, Heisenbergstraße 3, D-70569 Stuttgart, Germany \\ (Received 4 March 2014; revised manuscript received 17 April 2014; published 8 May 2014)
}

\begin{abstract}
The field distribution in the vortex lattice of a pure niobium single crystal with an external field applied along a three-fold axis has been investigated by the transverse-field muon-spin-rotation (TF- $\mu$ SR) technique over a wide range of temperatures and fields. The experimental data have been analyzed with Delrieu's solution for the form factor supplemented by phenomenological formulas for the parameters. This has enabled us to experimentally establish the temperatures and fields for Delrieu's, Ginzburg and Landau's, and Klein's regions of the vortex lattice. Using the numerical solution of the quasiclassical Eilenberger equation, the experimental results have been reasonably understood. They should apply to all clean BCS superconductors. Delrieu's analytical model supplemented by phenomenological formulas for its parameters is found to be reliable for analyzing TF- $\mu \mathrm{SR}$ experimental data for a substantial part of the mixed phase. Abrikosov's limit is contained in it.
\end{abstract}

DOI: 10.1103/PhysRevB.89.184503

PACS number(s): 75.25.-j, 74.20.Fg, 74.78.-w, 76.75.+i

\section{INTRODUCTION}

The physical properties of superconductors are usually described by the phenomenological Ginzburg-Landau (GL) theory $[1,2]$. For a type-II superconductor it predicts a mixed phase with a periodic variation of the magnetic induction in the form of a vortex lattice (VL), as first derived by Abrikosov [3]. For a simple superconductor the VL is characterized by three fields: the minimum field, the field at the saddle point of the field map, and the maximum field, which is found in the vortex cores. The minimum field lies at the center of the equilateral triangle formed by three nearest-neighbor vortex cores. This simple picture is believed to be valid, although the GL theory is theoretically justified only in the vicinity of $T_{\mathrm{c} 0}$ [4], the critical temperature at low field. However, because of its simplicity, it serves as a basis for data analysis of experiments performed in the whole mixed phase $[5,6]$.

Using an approximate solution of the microscopic BCSGor'kov equation [4], Delrieu discovered the minimum field in the vicinity of the upper critical field $B_{\mathrm{c} 2}$ at low temperature to be at the midpoint between two vortex cores [7]. Later on, solving numerically Eilenberger's equation [8], an analytical approximation to the BCS-Gor'kov's equation involving an integration over the magnitude of the electron wave vector, Klein found two field minima in the VL unit cell at intermediate temperature [9]. Consistent with Klein's results, nuclear magnetic resonance (NMR) experiments by Kung [10] on vanadium have detected a linear temperature dependence of the vortex-core field in a large temperature range towards zero temperature.

Here we present an exhaustive study of the field distribution in the $\mathrm{VL}$ of a pure niobium single crystal with the magnetic field $\mathbf{B}_{\text {ext }}$ applied along a crystallographic $\langle 111\rangle$ direction. The measurements have been done using the transversefield positive-muon-rotation (TF- $\mu \mathrm{SR}$ ) technique $[11,12]$. We have recently published a report which focuses on the $B_{\mathrm{c} 2}$ vicinity [13]. The present data have been analyzed with an expression for the form factor derived analytically by Delrieu. Notice that the form factor of Abrikosov is a limiting case of the former expression. Combining experimental and theoretical results, we have established the VL characteristics predicted by Abrikosov, Delrieu, and Klein in the proper parameter ranges. Our findings have been explained semiquantitatively using results obtained by solving numerically Eilenberger's equation assuming a cylindrical Fermi surface.

The organization of this paper is as follows. Section II introduces theoretical models for the VL field distribution. In Sec. III the sample is described, as well as the experimental conditions and the data analysis. Section IV displays typically measured field distributions. Section V discusses the VL characteristics derived from the present experimental and theoretical studies. We summarize the results obtained in this work in Sec. VI. Possible improvements of the data analysis and experimental conditions are mentioned. Some conclusions and perspectives are presented in Sec. VII.

The reader interested in only the characteristics of the VL for niobium derived from our measurements and their analysis should jump directly to Sec. V. We stress that they are expected to be found for all clean BCS superconductors.

\section{THEORETICAL BACKGROUND ON THE FIELD DISTRIBUTION IN THE VL OF NIOBIUM}

We shall first briefly describe the theories used to fit the $\mu \mathrm{SR}$ asymmetry time spectra. Then we shall present some computed field distributions.

We recall that a conventional triangular VL is observed when $\mathbf{B}_{\text {ext }}$ is applied along a three-fold axis as revealed by small-angle neutron scattering (SANS) [14-17]. In contrast to expectation, the VL field distribution is not described by the GL theory for $B_{\mathrm{ext}}$ in the $B_{\mathrm{c} 2}$ vicinity at low 


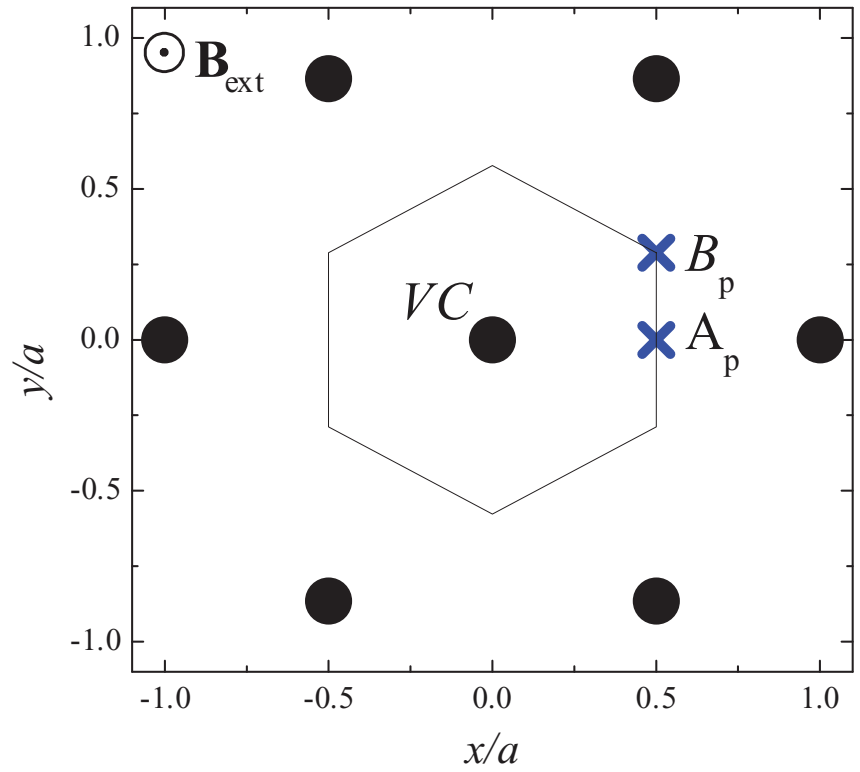

FIG. 1. (Color online) A triangular vortex lattice in the direct space. The external field $\mathbf{B}_{\text {ext }}$ is applied perpendicular to the vortex plane. The bullets illustrate vortex-core coordinates, and the crosses show two positions of particular interest in a unit cell, $A_{\mathrm{p}}$ and $B_{\mathrm{p}}$. In addition, we specify the position of one of the vortex-core centers with $V C$ nearby. The thin solid line represents the Wigner-Seitz cell. The position coordinates are scaled by the VL parameter $a$.

temperature $[13,18]$. On the other hand, the approximate Delrieu solution of the BCS-Gor'kov equation explains the measured distribution, but only above $0.6 \mathrm{~K}$ [13]. This is a temperature much lower than the zero-field critical temperature $T_{\mathrm{c} 0}=9.25 \mathrm{~K}$.

\section{A. Description of the available theories}

We illustrate in Fig. 1 an equilateral triangular VL. Three positions of special interest have been defined: the midpoint between two consecutive vortex cores $A_{\mathrm{p}}$ and the center of an equilateral triangle formed by three nearest-neighbor vortex cores $B_{\mathrm{p}}$. In addition, a vortex-core position is labeled $V C$.

The TF- $\mu$ SR technique gives access to the component field distribution along the $\mathbf{B}_{\text {ext }}$ direction if $B_{\text {ext }}$ is sufficiently large [12]. That is the case here. Labeling this direction as $Z$, $\tilde{D}_{\mathrm{c}}\left(B^{Z}\right)$ is measured. It can be computed from the real-space field map $B^{Z}(\mathbf{r})$ of the two-dimensional VL:

$$
\tilde{D}_{\mathrm{c}}\left(B^{Z}\right)=\int_{\text {u.c. }} \delta\left[B^{Z}(\mathbf{r})-B^{Z}\right] d^{2} \mathbf{r},
$$

where the integral extends over the VL unit cell. In terms of its Fourier components $B_{\mathbf{K}_{m, n}}^{Z}$, we have for the field map

$$
B^{Z}(\mathbf{r})=\sum_{\mathbf{K}_{m, n}} B_{\mathbf{K}_{m, n}}^{Z} \exp \left(i \mathbf{K}_{m, n} \cdot \mathbf{r}\right)
$$

where the sum is over the reciprocal space of the VL.

We need an expression for $B_{\mathbf{K}_{m, n}}^{Z}$, which is usually called the form factor in the SANS literature.

First of all, we note that our sample is in the clean limit [13]. So we do not need to consider the effect of impurities. We shall use two theories for the computation of $B_{\mathbf{K}_{m, n}}^{Z}$ that are approximations to the BCS-Gor'kov theory. Two types of approximations are to be considered: the common ones and the ones particular to a theory. Common is the semiclassical approximation. Here the hypothesis is made that the spacing between the Landau levels is small in comparison to the sum of their thermal and collision broadenings [19]. This is expected to be valid for niobium down to $\approx 0.04 \mathrm{~K}$ [20]. To get quantitative predictions to compare with experimental data, we need a simple, but still realistic, Fermi surface. Delrieu's solution of the BCS-Gor'kov equation assumes a spherical Fermi surface. For an extensive numerical study of Eilenberger's equation such as presented here, a cylindrical Fermi surface is a natural choice. This is partly because of computational convenience and is believed to be enough for the present purpose of constructing the phase diagram. Its essential features and other properties in this paper do not change when a three-dimensional Fermi surface model is used; only the $\kappa_{\mathrm{GL}}$ value changes. We can translate and interpret the $\kappa_{\mathrm{GL}}$ values between the two-dimensional and three-dimensional cases. As for more realistic Fermi surface models, some of us have the experience to use a realistic three-dimensional Fermi surface model calculated by band theory for niobium [21]. In order to evaluate the subtle vortex lattice orientational changes for $\mathbf{B}_{\text {ext }} \|\langle 001\rangle$ we definitely need to have a realistic Fermi surface model. For the present purposes the cylindrical Fermi surface model is believed to be enough.

Next, for completeness we summarize the work of Delrieu. He neglected the spatial dependence of the order parameter $\Delta(\mathbf{r})$. While this approximation is reasonable in the vicinity of $B_{\mathrm{c} 2}$, it should break down when approaching the lower critical field. He derived $B_{\mathbf{K}_{m, n}}^{Z}=f_{m, h}(\tilde{a}, \tilde{b}, \tilde{c})$. The function $f_{m, h}$ can be found elsewhere $[7,20]$. Here $\tilde{a}$ has the dimension of a field:

$$
\tilde{a}=-\frac{\mu_{0} N_{0} \Delta_{0}^{2}}{2 \overline{B^{Z}}} \tilde{c} .
$$

In the region of validity of Delrieu's approximation, $\overline{B^{Z}(\mathbf{r})} \equiv$ $\overline{B^{Z}} \simeq B_{\text {ext }}$. The parameter $\tilde{a}$ does not influence the shape of $B_{\mathbf{K}_{m, n}}^{Z}$ and $\tilde{D}_{\mathrm{c}}\left(B^{Z}\right)$. It only gives its scale. It is proportional to the density of state at the Fermi level in the normal metal $N_{0}$ (per spin, volume, and energy) and the quantity $\Delta_{0}^{2}=\overline{|\Delta(\mathbf{r})|^{2}}$ $\left[\overline{|\Delta(\mathbf{r})|^{2}}\right.$ is the spatial average of $\left.|\Delta(\mathbf{r})|^{2}\right]$ and is inversely proportional to the average field $\overline{B^{Z}}$. The dimensionless parameters $\tilde{b}$ and $\tilde{c}$ determine the shape of $\tilde{D}_{\mathrm{c}}\left(B^{Z}\right)$ and are expressed in terms of the ratios of three length scales: $\tilde{b}=\left[\Lambda /\left(\pi \xi^{B}\right)\right]^{2}$ and $\tilde{c}=\Lambda / \xi^{T}$. Here, $\Lambda=\left[\Phi_{0} /\left(2 \pi \overline{B^{Z}}\right)\right]^{1 / 2}$ is a length parameter proportional to the intervortex distance. The field- and temperature-dependent length scale $\xi^{B}=$ $\hbar v_{\mathrm{F}} /\left(\pi \Delta_{0}\right)$ diverges near $B_{\mathrm{c} 2}$, while $\xi^{T}=\hbar v_{\mathrm{F}} /\left(2 \pi k_{\mathrm{B}} T\right)$. We have introduced the Fermi velocity $v_{\mathrm{F}}$. It is easily found that

$$
\tilde{b}=\frac{1}{\pi^{2}} \frac{\xi_{\mathrm{GL}}^{2}(T)}{\xi_{0}^{2}(T)} \frac{1-b}{b},
$$

where $b=B_{\text {ext }} / B_{c 2}(T)$ is the reduced field, $\xi_{\mathrm{GL}}$ is the GL coherence length, and $\xi_{0}$ is the Pippard-BCS coherence length [20]. To derive Eq. (4) two phenomenological formulas expected to be valid for conventional superconductors have 
been used:

$$
\begin{gathered}
\Delta_{0}=\Delta_{0}(0) \sqrt{1-b} \sqrt{1-\tau^{2}}, \\
B_{\mathrm{c} 2}(T)=B_{\mathrm{c} 2}(0)\left(1-\tau^{2}\right),
\end{gathered}
$$

where $\quad \tau=T / T_{\mathrm{c} 0}$. Since $\quad \frac{\xi_{\mathrm{GL}}^{2}(T)}{\xi_{0}^{2}(T)}=\frac{\xi_{\mathrm{GL}}^{2}(0)}{\xi_{0}^{2}(0)} \quad$ and $\xi_{\mathrm{GL}}(0) \simeq \xi_{0}(0) / 0.96$ in the clean limit $[1]$,

$$
\tilde{b}=0.110 \frac{1-b}{b} \text {. }
$$

Hence, $\tilde{b}$ only depends on $b$. The parameter $\tilde{c}$ can be expressed in terms of $v_{\mathrm{F}}[20]$ :

$$
\tilde{c}=\frac{\sqrt{\Phi_{0} 2 \pi} k_{\mathrm{B}} T}{\sqrt{\overline{B^{Z}}} \hbar v_{\mathrm{F}}},
$$

where $\Phi_{0}$ is the magnetic flux quantum. Interestingly, $N_{0} \Delta_{0}^{2}$ in Eq. (3) is the condensation energy $E_{\mathrm{c}}$ [13]. Hence, according to Eq. (5),

$$
E_{\mathrm{c}}=E_{\mathrm{c}}(0)(1-b)\left(1-\tau^{2}\right) .
$$

This means that $B_{\mathbf{K}_{m, n}}^{Z}$ from Delrieu's solution supplemented by phenomenological formulas for the parameters depends on two material parameters: $v_{\mathrm{F}}$ and $E_{\mathrm{c}}(0)$, the second parameter being involved in only the scaling of the field. Introducing the unitless field,

$$
b_{\mathrm{N}}^{Z}=\frac{B^{Z}-B_{\mathrm{sad}}}{B_{\mathrm{vc}}-B_{\mathrm{sad}}},
$$

where $B_{\mathrm{sad}}$ and $B_{\mathrm{vc}}$ are the saddle-point and vortex-core fields discussed at length in Sec. II B. While $B_{\mathrm{vc}}$ is always the field at a vortex-core center, the position of $B_{\text {sad }}$ may change as described in Sec. VB. The unitless component field distribution $D_{\mathrm{c}}\left(b_{\mathrm{N}}^{Z}\right)$ only depends on $v_{\mathrm{F}}$. However, for the computation of a measured TF- $\mu$ SR asymmetry time spectrum we need $\tilde{D}_{\mathrm{c}}\left(B^{Z}\right)$ rather than $D_{\mathrm{c}}\left(b_{\mathrm{N}}^{Z}\right)$. Since $D_{\mathrm{c}}\left(b_{\mathrm{N}}^{Z}\right) d b_{\mathrm{N}}^{Z}=$ $\tilde{D}_{\mathrm{c}}\left(B^{Z}\right) d B^{Z}$

$$
\tilde{D}_{\mathrm{c}}\left(B^{Z}\right)=\frac{1}{B_{\mathrm{vc}}-B_{\mathrm{sad}}} D_{\mathrm{c}}\left(b_{\mathrm{N}}^{Z}\right) .
$$

Hence, as expected, $\tilde{D}_{\mathrm{c}}\left(B^{Z}\right)$ depends on two materials parameters, namely, $v_{\mathrm{F}}$ and $E_{\mathrm{c}}(0)$, and $D_{\mathrm{c}}\left(b_{\mathrm{N}}^{Z}\right)$ depends only on $v_{\mathrm{F}}$.

Since the $B_{\mathbf{K}_{m, n}}^{Z}$ analytical Delrieu's solution derives from an approximation to the BCS-Gor'kov theory supposed to be valid only in the $B_{\mathrm{c} 2}$ vicinity, we need a method to compute $B_{\mathbf{K}_{m n}}^{Z}$ for the whole VL. In addition, if possible, it would be nice not to rely on phenomenological formulas for the physical parameters. Eilenberger's equation for the thermal Green's function fits our purpose. Eilenberger introduced Green's functions that result from Gor'kov's functions integrated over the magnitude of the electron wave vector [8]. These former functions follow transport-like equations suitable for numerical calculations as first shown by Klein [9]. Supplemented by the self-consistent equations for the gap function and vector potential, here we have directly computed $B^{Z}(\mathbf{r})$ normalized by $B_{\mathrm{c} 2}(0)$, a quantity directly observable.

The integration over the magnitude of the wave vector introduces an approximation which is valid when $k_{\mathrm{F}} \xi_{0} \gg 1$, where $k_{\mathrm{F}}$ is the Fermi wave vector. Since $1 / k_{\mathrm{F}}$ is of the order of the niobium lattice parameter and $\xi_{0} \simeq 27 \mathrm{~nm}$ [20], the condition $k_{\mathrm{F}} \xi_{0} \gg 1$ is clearly fulfilled. Following Brandt's method for solving the GL equations [22], Eilenberger's equation is currently solved by taking advantage of the periodicity of the VL [23]. Nicely enough, $B^{Z}(\mathbf{r}) / B_{\mathrm{c} 2}(0)$ depends only on one single material parameter: the GL parameter $\kappa_{\mathrm{GL}}=\lambda / \xi_{\mathrm{GL}}$, where $\lambda$ is the London penetration depth. This should be compared to $B_{\mathbf{K}_{m, n}}^{Z} / \tilde{a}$ from Delrieu, which also depends on one single parameter, but $v_{\mathrm{F}}$ rather than $\kappa_{\mathrm{GL}}$. These two parameters are related [9].

\section{B. Characteristics of field distributions}

We compare $D_{\mathrm{c}}\left(b_{\mathrm{N}}^{Z}\right)$ for some selected $\left(T, B_{\text {ext }}\right)$ values computed from the analytical Delrieu theory in Fig. 2. We take the material parameter valid for our niobium sample [13]: $v_{\mathrm{F}}=2.0 \times 10^{5} \mathrm{~m} / \mathrm{s}$. We recall that unitless $D_{\mathrm{c}}\left(b_{\mathrm{N}}^{Z}\right)$ only depends on $v_{\mathrm{F}}$. We recall that $T_{\mathrm{c} 0}=9.25 \mathrm{~K}$ and $B_{\mathrm{c} 2}(0)=$ 0.43 T. As expected and clearly seen in Fig. 2, a distribution is characterized by three fields: its minimum field $B_{\min }$, a saddle-point field $B_{\text {sad }}$ in the field map for which $D_{\mathrm{c}}\left(b_{\mathrm{N}}^{Z}\right)$ displays a maximum, and $B_{\mathrm{vc}}$, which is the field in the center of a vortex core, i.e., the maximum field in $D_{\mathrm{c}}\left(b_{\mathrm{N}}^{Z}\right)$. Note that two features of a distribution are strongly dependent on the $\left(T, B_{\text {ext }}\right)$ values: the shape of the high-field tail and the distance between $B_{\text {min }}$ and $B_{\text {sad }}$. As shown previously [13], the observation of a linear high-field tail for large $B_{\text {ext }}$ and low $T$, clearly seen for the fourth distribution from the top, is a signature in those experimental conditions of the pronounced conical shape of the field variation around the vortex cores. This results from the partial Cooper pair diffraction on the vortex cores. We find

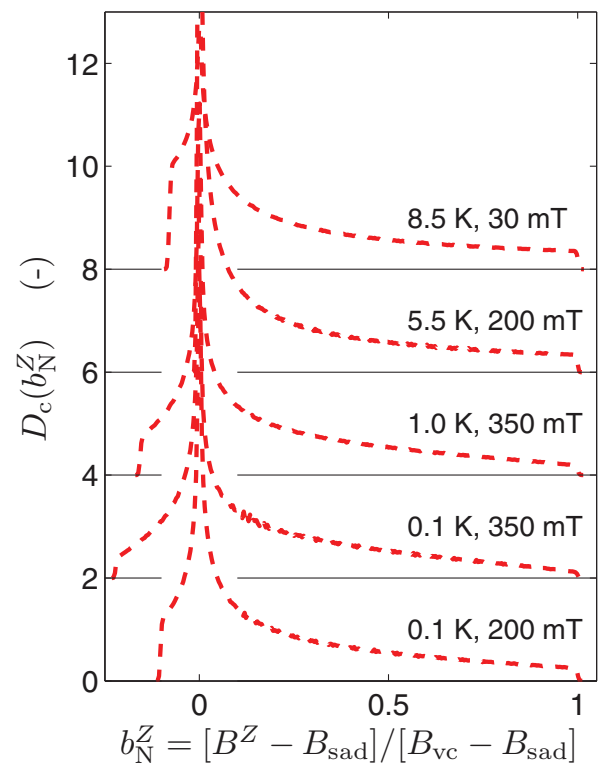

FIG. 2. (Color online) Component field distributions $D_{\mathrm{c}}\left(b_{\mathrm{N}}^{Z}\right)$ computed with Delrieu's model assuming $v_{\mathrm{F}}=2.0 \times 10^{5} \mathrm{~m} / \mathrm{s}$. Five cases are considered: from top to bottom, in the $B_{\mathrm{c} 2}(T)$ vicinity at four temperatures, i.e., $T=8.5,5.5,1.0$, and $0.1 \mathrm{~K}$, and at low temperature and field but still in the mixed phase, i.e., $T=0.1 \mathrm{~K}$ with $B_{\text {ext }}=200 \mathrm{mT}$. 
it convenient to measure the distance between $B_{\min }$ and $B_{\text {sad }}$ with the following unitless normalized ratio:

$$
\delta B_{\mathrm{sad}, \text { min }}^{\mathrm{n}}=-\frac{B_{\mathrm{min}}-B_{\mathrm{sad}}}{B_{\mathrm{vc}}-B_{\mathrm{sad}}} .
$$

The first three distributions from the top of Fig. 2 are for $D_{\mathrm{c}}\left(b_{\mathrm{N}}^{Z}\right)$ with $B_{\text {ext }}$ in the $B_{\mathrm{c} 2}(T)$ vicinity. A $\delta B_{\text {sad,min }}^{\mathrm{n}}$ minimum is predicted around $T=5.5 \mathrm{~K}$. This should easily be observed experimentally. However, because of the Gaussian smearing discussed in the next section, $\delta B_{\text {sad,min }}^{\mathrm{n}}$ is not expected to be as small as predicted. We postpone the discussion of its physical meaning to Sec. V. A close look at the last two $D_{\mathrm{c}}\left(b_{\mathrm{N}}^{Z}\right)$ from the top of Fig. 2 illustrates the effect of the field intensity at low temperature. An exotic $D_{\mathrm{c}}\left(b_{\mathrm{N}}^{Z}\right)$ is only predicted for a sufficiently large $B_{\text {ext }}$.

\section{EXPERIMENT}

Here the sample is described, along with the experimental conditions and the data analysis.

The TF- $\mu$ SR measurements reported here have been performed on the single crystal described in Ref. [13]. The small $B_{\mathrm{c} 2}=430$ (2) mT testifies of its high quality and purity, as well as the lack of difference between the distributions measured with the zero-field-cooled and field-cooled procedures at $1.5 \mathrm{~K}$ under $B_{\text {ext }}=360 \mathrm{mT}$.

The new TF- $\mu \mathrm{SR}$ measurements described here have again been performed at the Swiss Muon Source $(\mathrm{S} \mu \mathrm{S})$, Paul Scherrer Institute (PSI), Switzerland, using the general purpose spectrometer (GPS) and Dolly spectrometers for $T \geqslant 1.6 \mathrm{~K}$. Measurements for $T<1.6 \mathrm{~K}$ have been conducted on the low-temperature facility (LTF) spectrometer.

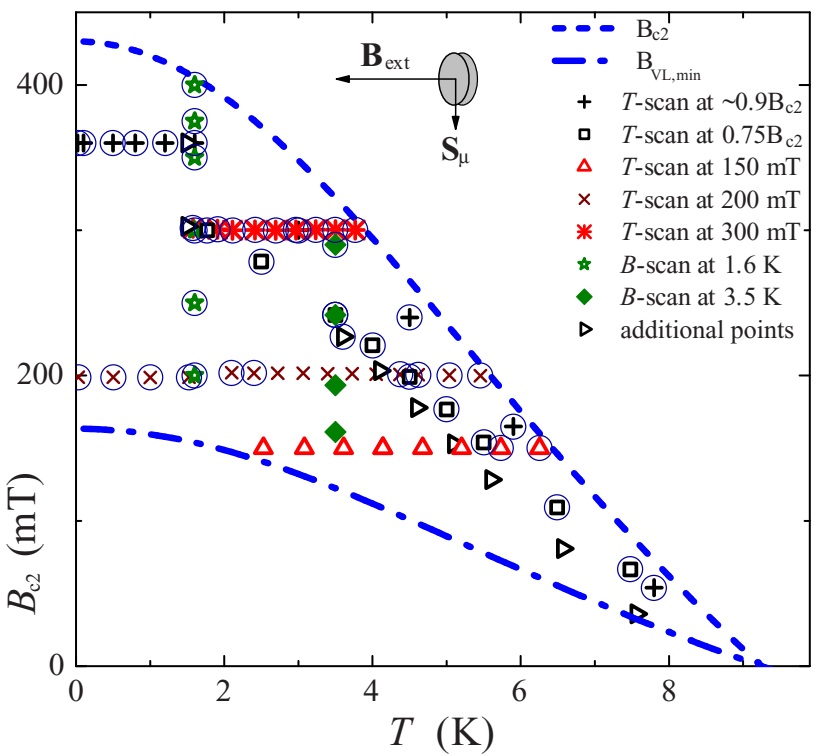

FIG. 3. (Color online) Temperatures and fields at which measurements have been performed on the niobium single crystal. The geometry for a measurement is depicted by the pictogram: the directions of $\mathbf{B}_{\text {ext }} \|\langle 111\rangle$ and of the initial muon beam polarization $\mathbf{S}_{\mu}$ are given. The $B_{\mathrm{VL}, \min }(T)$ and $B_{\mathrm{c} 2}(T)$ lines have been determined as explained in the main text. The points at which the form factor from Delrieu provides a proper description of the experimental $\mu \mathrm{SR}$ data are encircled.

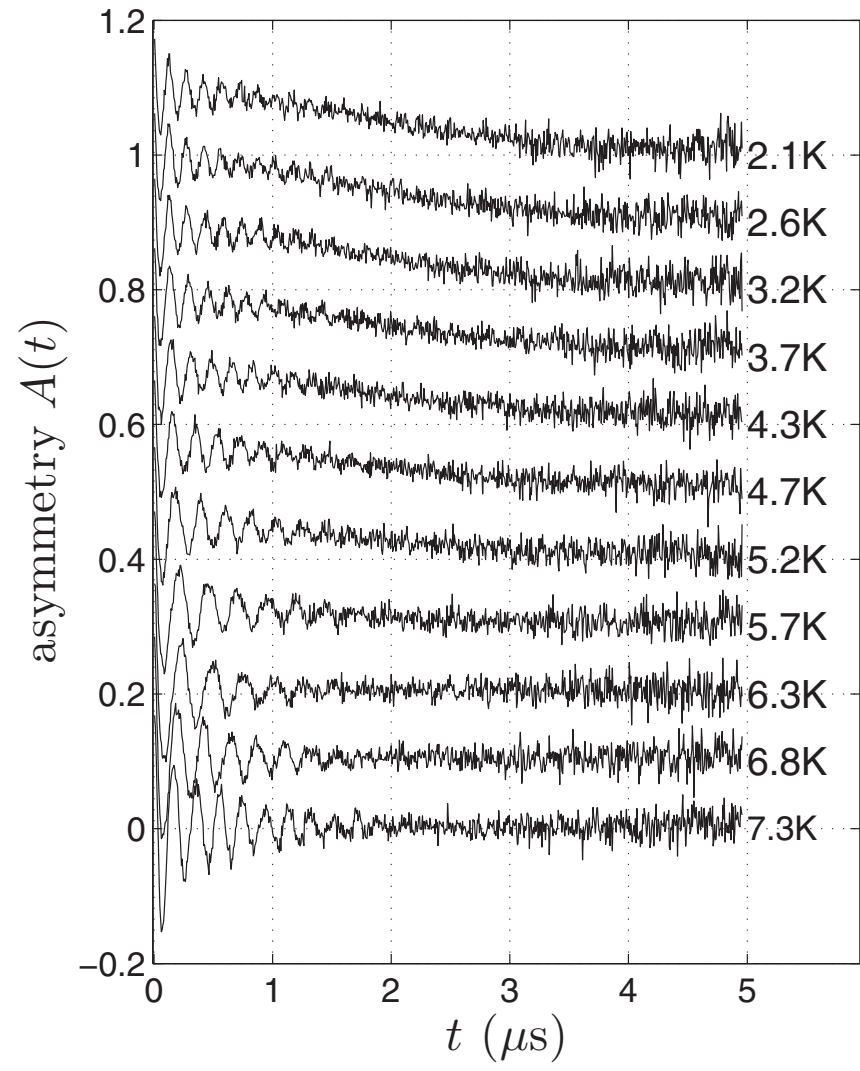

FIG. 4. Some asymmetry time spectra for our niobium single crystal with $\mathbf{B}_{\text {ext }} \|\langle 111\rangle$ as a function of temperature at $B_{\text {ext }}=50 \mathrm{mT}$.

Our niobium sample is a single-crystal disk of $13 \mathrm{~mm}$ diameter and $2 \mathrm{~mm}$ thickness with a three-fold axis oriented normal to the disk. In Fig. 3 we specify the values of the temperatures and fields for which measurements have been done. Five temperature scans have been performed at $B_{\text {ext }}=$ 300,200 , and $150 \mathrm{mT}, 0.75 B_{\mathrm{c} 2}$, and $\sim 0.9 B_{\mathrm{c} 2}$. In addition, we report two field scans at 1.6 and $3.5 \mathrm{~K}$. The $B_{\mathrm{c} 2}(T)$ line has been determined previously [13]. In addition to the traditional VL and Meissner phases, one needs to consider the intermediate state [24]. The VL phase is characterized by a single damped oscillation centered around zero as seen for $T=7.3$ and $6.8 \mathrm{~K}$ in Fig. 4. The amplitude of the oscillating signal at time $t=0$ is proportional to the fraction of the niobium sample in the $\mathrm{VL}$ state. When cooling down to $5.7 \mathrm{~K}$, the oscillation is no longer centered around zero, and its amplitude is reduced. The signals below $5.7 \mathrm{~K}$ represent the sum of oscillating and Kubo-Toyabetype components. This indicates that the sample has left the mixed phase and part of it is in a zero-field condition due to Meissner screening. Performing a few series of measurements as reported in Fig. 4, we have determined the $B_{\mathrm{VL}, \min }(T)$ line shown in Fig. 3. For $B_{\text {ext }}>B_{\mathrm{VL} \text {, min }}(T)$ the sample is certainly in the mixed phase. We shall use this conservative estimate.

The analysis of the asymmetry time spectra has been done following the method explained in Ref. [13]. Here we would like to stress three points. We do fit the time spectra and not the component field distributions, which are only computed for display purposes. Such a distribution is denoted $\tilde{D}_{\mathrm{c}}^{\exp }\left(B^{Z}\right)$. The difference between $\tilde{D}_{\mathrm{c}}^{\exp }\left(B^{Z}\right)$ and $\tilde{D}_{\mathrm{c}}\left(B^{Z}\right)$ arises from the contributions of the nuclear ${ }^{93} \mathrm{Nb}$ magnetic moments 
and the VL disorder to the field distribution at the muon site. These contributions are taken into account in the fits by a single Gaussian function [25]. This leads to a Gaussian smearing of $\tilde{D}_{\mathrm{c}}\left(B^{Z}\right)$. The influence of disorder is relatively modest in the high-field part of a distribution [26]. Finally, it has been shown previously that no effect of the muon diffusion on the measured $\tilde{D}_{\mathrm{c}}^{\exp }\left(B^{Z}\right)$ is expected [13].

Data analysis with Delrieu's model is exceedingly time consuming. The computation of $\tilde{D}_{\mathrm{c}}\left(B^{Z}\right)$ may take a few minutes. Since a large number of iterations are needed for fitting a single asymmetry spectrum, an analysis would take many hours. In order to accelerate the analysis we have first computed $B_{\mathbf{K}_{m, h}}^{Z}\left(\tilde{b}_{i}, \tilde{c}_{j}\right)$ setting $\tilde{a}=1$ since it is only a multiplicative factor. We have taken $-30 \leqslant m \leqslant 30$ and $-30 \leqslant h \leqslant 30$ and a discrete set of $\tilde{b}_{i}$ and $\tilde{c}_{j}$ parameters, i.e., $\tilde{b}_{i}=10^{n_{i}}$ and $\tilde{c}_{j}=$ $10^{n_{j}}$, with $n_{\alpha}=-3+0.05 \times(\alpha-1)(\alpha$ stands for $i$ or $j)$. The indices $i=1,2, \ldots, 81$ and $j=1,2, \ldots, 101$ correspond to $0.001 \leqslant \tilde{b} \leqslant 10$ and $0.001 \leqslant \tilde{c} \leqslant 100$. Because $B_{\mathbf{K}_{m, h}}^{Z}$ is a continuous function of its variables, in the fitting procedure the actual values of $B_{\mathbf{K}_{m, h}}^{Z}(\tilde{a}, \tilde{b}, \tilde{c})$ were evaluated by interpolation from the precalculated values of $\tilde{a} \times B_{\mathbf{K}_{m, h}}^{Z}\left(\tilde{b}_{i}, \tilde{c}_{j}\right)$ (fourdimensional matrix). A quadratic interpolation has been used to avoid zero second-order derivatives during the $\chi^{2}$ minimization. With this method an evaluation of asymmetry time spectrum or $\tilde{D}_{\mathrm{c}}\left(B^{Z}\right)$ can be performed within a fraction of second.

\section{TYPICAL MEASURED FIELD DISTRIBUTIONS}

In this section typical measured field distributions $D_{\mathrm{c}}^{\exp }\left(b_{\mathrm{N}}^{Z}\right)$ are displayed. The curves result from a combined fit of the measured asymmetry time spectra to Delrieu's theory with $v_{\mathrm{F}}$ as a global fitting parameter. The parameters extracted from it are discussed in Sec. V.

We start by considering the $0.75 B_{\mathrm{c} 2}(T)$ temperature scan. As seen from Fig. 3, it probes the VL from near $T_{\mathrm{c} 0}$ down to low temperature, i.e., from 7.5 to $1.77 \mathrm{~K}$. Figure 5 illustrates some
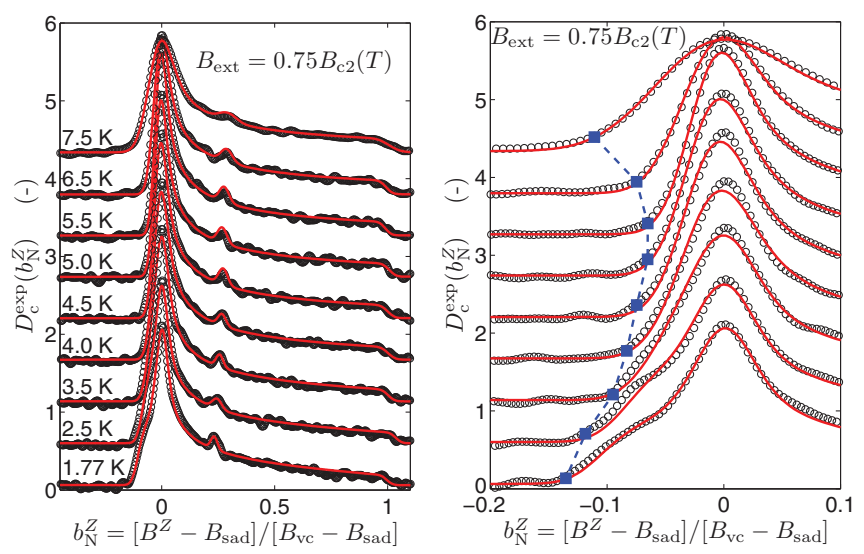

FIG. 5. (Color online) Some $D_{\mathrm{c}}^{\text {exp }}\left(b_{\mathrm{N}}^{Z}\right)$ distributions as a function of temperature for $B_{\mathrm{ext}}=0.75 B_{\mathrm{c} 2}(T)$. The data and curves have been shifted vertically for better visualization. For each $D_{\mathrm{c}}^{\exp }\left(b_{\mathrm{N}}^{Z}\right)$ the weak intensity peak for $b_{\mathrm{N}}^{Z}$ slightly larger than 0.2 arises from the contribution of the background (sample holder and cryostat walls) to the distribution. The right panel focuses on the low-field regime. The solid lines result from fits to Delrieu's theory as described in Sec. II. The dashed line links the $B_{\min }$ values.
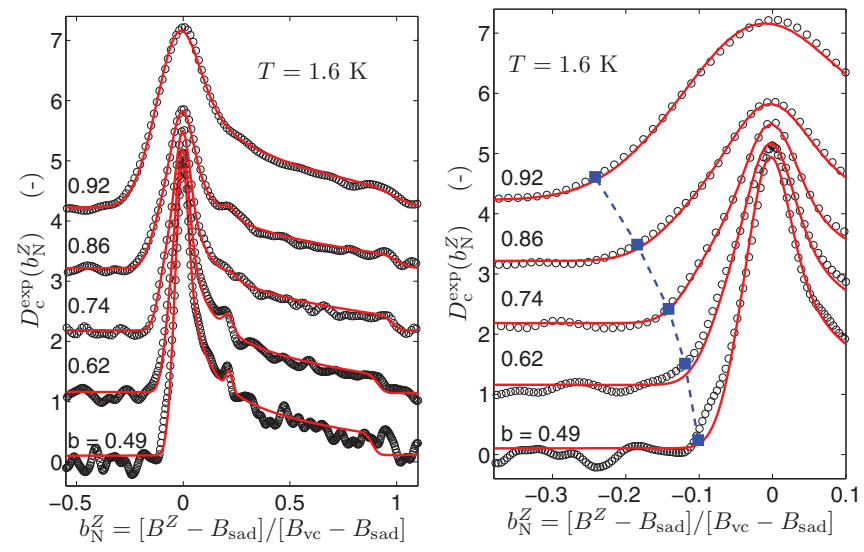

FIG. 6. (Color online) A selection of $D_{\mathrm{c}}^{\exp }\left(b_{\mathrm{N}}^{Z}\right)$ distributions as a function of the reduced field $b=B_{\mathrm{ext}} / B_{\mathrm{c} 2}(1.6 \mathrm{~K})$. The data and curves have been shifted vertically for better visualization. For each $D_{\mathrm{c}}^{\exp }\left(b_{\mathrm{N}}^{Z}\right)$ the weak-intensity peak for $b_{\mathrm{N}}^{Z} \simeq 0.2$ arises from the contribution of the background (sample holder and cryostat walls) to the distribution. The limited statistic for the $b=0.49$ distribution explains its rather noisy nature. The right panel focuses on the low-field regime. The solid lines result from fits to Delrieu's theory as described in Sec. II. The dashed line links the $B_{\min }$ values.

$D_{\mathrm{c}}^{\text {exp }}\left(b_{\mathrm{N}}^{Z}\right)$. Delrieu's theory provides a good description. This is indicated in Fig. 3 by encircling the symbols which specify the temperatures and fields of the scan. The determination of $B_{\min }$ at high temperature is not very precise due to Gaussian smearing. However, from Fig. 5 it is quite clear that $\delta B_{\text {sad,min }}^{\mathrm{n}}$ is minimum around $5.0-5.5 \mathrm{~K}$.

Some $D_{\mathrm{c}}^{\exp }\left(b_{\mathrm{N}}^{Z}\right)$ from the field scan at $1.6 \mathrm{~K}$ are shown in Fig. 6. Here a smooth $\delta B_{\text {sad,min }}^{\mathrm{n}}$ increase is observed as $B_{\mathrm{c} 2}$ is approached. Again, Delrieu provides a good description.

Two $D_{\mathrm{c}}^{\exp }\left(b_{\mathrm{N}}^{Z}\right)$ are displayed in Fig. 7 for $T \simeq 6.5 \mathrm{~K}$. The remarkable feature here is that $\delta B_{\text {sad,min }}^{\mathrm{n}}$ is about the same for the two distributions, although the reduced field $b$ is clearly different. This is in contrast to the two previous sets of
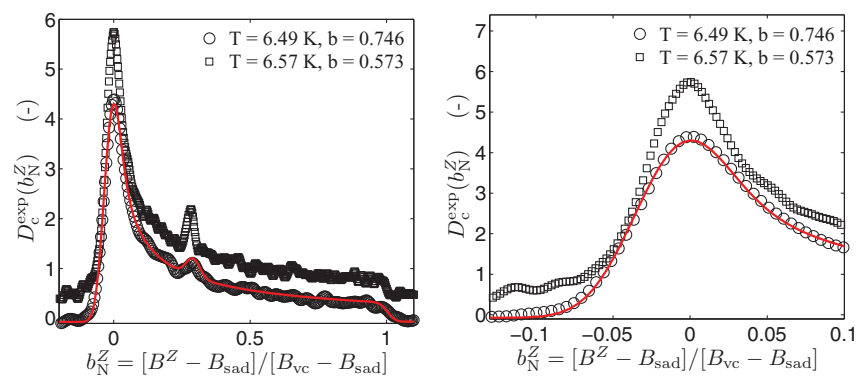

FIG. 7. (Color online) Two $D_{\mathrm{c}}^{\exp }\left(b_{\mathrm{N}}^{Z}\right)$ distributions for $T \simeq 6.5 \mathrm{~K}$ at two different fields. The data and curves have been shifted vertically for better visualization. The peaks around $b_{\mathrm{N}}^{Z} \simeq 0.3$ arise from the contributions of the background (sample holder and cryostat walls) to the distributions. The right panel focuses on the low-field regime. The value of the reduced field $b=B_{\mathrm{ext}} / B_{\mathrm{c} 2}(1.6 \mathrm{~K})$ is indicated for each $D_{\mathrm{c}}^{\text {exp }}\left(b_{\mathrm{N}}^{Z}\right)$. A larger contribution of the VL disorder to the higher-field distribution is seen by the reduction of the probability at $B_{\text {sad. }}$. The solid lines result from fits to Delrieu's theory as described in Sec. II for $T=6.49 \mathrm{~K}$ and $b=0.746$. That theory does not fit the data for $T=6.57 \mathrm{~K}$ and $b=0.573$. 
distributions for which $\delta B_{\text {sad,min }}^{\mathrm{n}}$ changes as a function of field or temperature. Delrieu's model is unable to account for the data at the lowest $B_{\text {ext }}$ value.

\section{CHARACTERISTICS OF THE MIXED PHASE OF NIOBIUM}

Here the physical properties of niobium and its VL deduced from our measured $\mu \mathrm{SR}$ data are discussed. We shall first consider the parameters extracted from the global fit of the asymmetry time spectra with Delrieu's approximation for the form factor. The validity regime of the approximation will be determined. Then the properties of the three characteristic fields of the VL will be analyzed with the numerical solution of Eilenberger's theory. Finally, combining our experimental results and Eilenberger's theory, the field map of the VL will be established.

\section{A. VL parameters and region of validity of Delrieu's approximation}

We have recorded TF- $\mu$ SR asymmetry time spectra for the $\mathrm{VL}$ of niobium for a large range of $\left(T, B_{\text {ext }}\right)$ values. From a global fit of the spectra with Delrieu's approximation for the form factor we deduce $v_{\mathrm{F}}=2.0(2) \times 10^{5} \mathrm{~m} / \mathrm{s}$, in agreement with our previous estimate [13]. From the measured $\tilde{a}$ and $\tilde{c}$ parameters and Eq. (3) the condensation energy $E_{\mathrm{c}}=N_{0} \Delta_{0}^{2}$ is determined. As an example, in Fig. 8 we show the field dependence of $E_{\mathrm{c}}=-2 \overline{B^{Z}} \tilde{a} / \mu_{0} \tilde{c}$ at $T=1.6 \mathrm{~K}$. The linear field dependence of $\Delta_{0}^{2}$ given by Eq. (5) is found to be a reasonable approximation. This is again consistent with the results of our analysis of spectra previously taken in the vicinity of $B_{\mathrm{c} 2}(T)$.

From the analysis of an asymmetry time spectrum recorded near $T_{\mathrm{c} 0}$ with the GL theory we recall that $\kappa_{\mathrm{GL}}=0.89$ (1) [20].

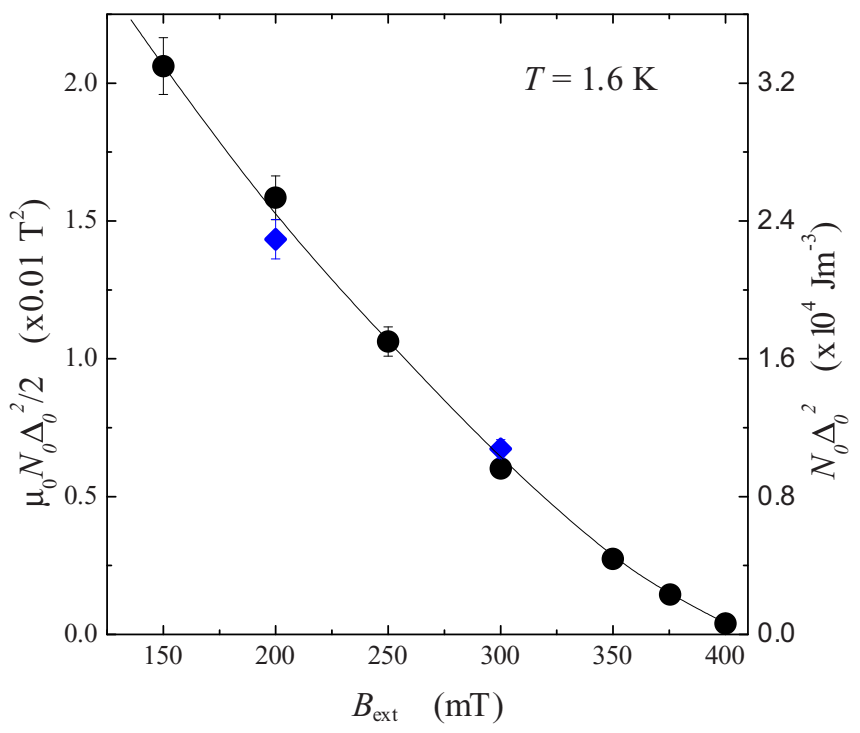

FIG. 8. (Color online) Condensation energy $E_{\mathrm{c}}=N_{0} \Delta_{0}^{2}$ and $\mu_{0} N_{0} \Delta_{0}^{2} / 2$ as a function of $B_{\text {ext }}$. The values extracted from the field scan at $1.6 \mathrm{~K}$ are represented as circles, while diamonds correspond to the two temperature scans at 200 and $300 \mathrm{mT}$. The solid line is a guide to the eye.
From a close look at Fig. 3 we infer that Delrieu's solution for the form factor has a relatively large validity range for niobium; that is, it is valid not only in the immediate vicinity of $B_{\mathrm{c} 2}(T)$ but also for $0.6 \leqslant T \leqslant 2.5 \mathrm{~K}$ with $0.2 \mathrm{~T} \leqslant B_{\text {ext }}<B_{\mathrm{c} 2}$. The lower temperature bound was previously given [13]. Since this solution also includes Abrikosov's result and it is numerically feasible to use it in a fit procedure, it should be seriously considered for the analysis of $\mathrm{TF}-\mu \mathrm{SR}$ data as a reliable alternative to a pure GL fit for clean $s$-wave superconductors [22,27].

\section{B. VL characteristic fields, field distributions, and physical origins}

Having finished the analysis of the experimental data for various fields and temperatures with Delrieu's theory, we now consider those from the viewpoint of Eilenberger's theory. Hence, we can discuss the whole mixed phase, not only the vicinity of $B_{\mathrm{c} 2}(T)$. As already mentioned, Klein first calculated the detailed field profiles in the mixed state of niobium by solving Eilenberger's equation [9]. Here, based on a numerical algorithm explained in Refs. [23,28-31], we have calculated $B^{Z}(\mathbf{r})$ within a VL unit cell under periodic boundary conditions for various $B_{\text {ext }}$ and $T$ appropriate for the present experimental situations. It will turn out later that $\kappa_{\mathrm{GL}}=1.8$ best describes the experimental data; thus all the following computations have been performed using this value. From this information a variety of physical quantities directly related to the present experiments can be deduced, that is, the field distribution $\tilde{D}_{\mathrm{c}}\left(B^{Z}\right)$ and therefore the three characteristic field values $B_{\min }, B_{\mathrm{sad}}$, and $B_{\mathrm{vc}}$ and their locations within a unit cell.

In order to understand the three possible field patterns, namely, those predicted by Ginzburg and Landau, Klein (KL), and Delrieu (DL), which we will identify through the analysis,

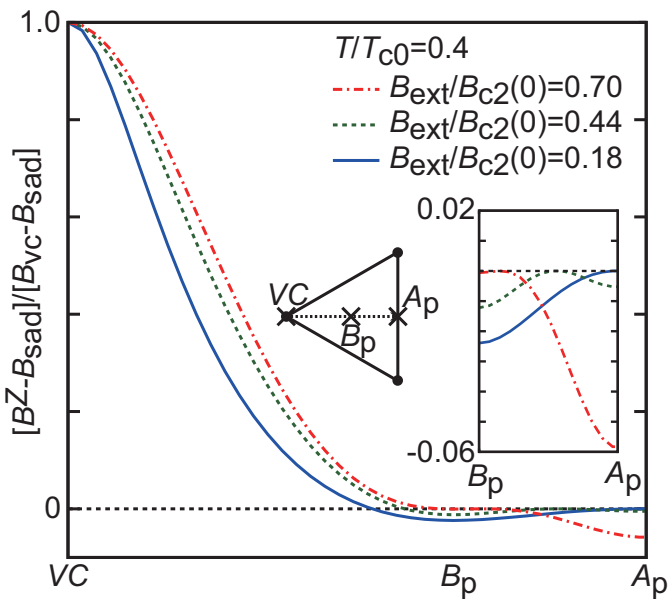

FIG. 9. (Color online) Results from Eilenberger's theory for three field profiles along the vortex-core- $A_{\mathrm{p}}$ path, i.e., along $V C-A_{\mathrm{p}}$ via $B_{\mathrm{p}}$ in the direct space. The ratio value $B_{\mathrm{ext}} / B_{\mathrm{c} 2}(0)=0.18$ corresponds to GL, $B_{\text {ext }} / B_{\mathrm{c} 2}(0)=0.44$ to $\mathrm{KL}$, and $B_{\mathrm{ext}} / B_{\mathrm{c} 2}(0)=$ 0.70 to DL. We have chosen $T / T_{\mathrm{c} 0}=0.4$. One of the insets recalls the definition of positions of interest in the unit cell, and the other inset magnifies the field profiles between $B_{\mathrm{p}}$ and $A_{\mathrm{p}}$. 


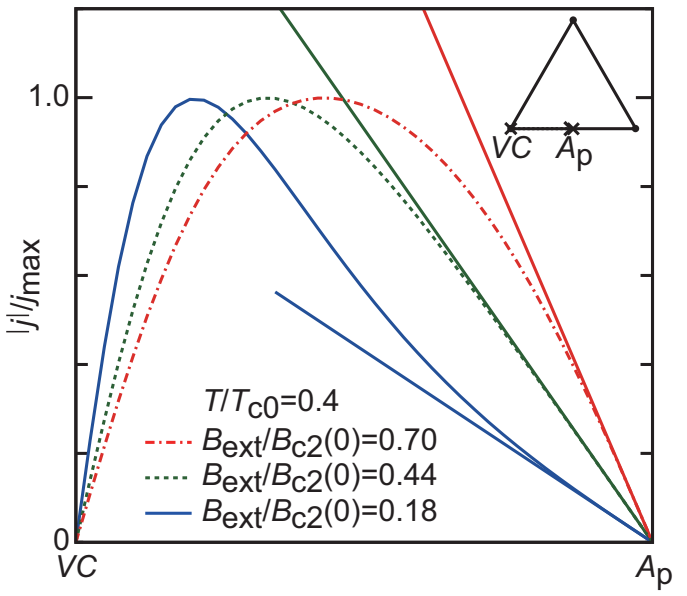

FIG. 10. (Color online) Three current profiles along the $V C-$ $A_{\mathrm{p}}$ path in the direct space. The parameters are the same as in Fig. 9. The currents are normalized to their maximum values. Note the changes in the slope at $A_{\mathrm{p}}$ and in the maximum positions for the three profiles.

we first show related field profiles for the three cases in Fig. 9. We find the following.

(1) The GL field profile for $B_{\mathrm{ext}} / B_{\mathrm{c} 2}(0)=0.18: B_{\min }$ is located at point $B_{\mathrm{p}}$ and $B_{\mathrm{sad}}$ at $A_{\mathrm{p}}$ in the unit cell. The lowest edge of $\tilde{D}_{\mathrm{c}}\left(B^{Z}\right)$ occurs at $B_{\mathrm{p}}$.

(2) The DL field profile for $B_{\mathrm{ext}} / B_{\mathrm{c} 2}(0)=0.70: B_{\min }$ is located at point $A_{\mathrm{p}}$, while $B_{\text {sad }}$ is located at point $B_{\mathrm{p}}$ in the unit cell. The lowest edge of $\tilde{D}_{\mathrm{c}}\left(B^{Z}\right)$ occurs at $A_{\mathrm{p}}$.

(3) The KL field profile for $B_{\text {ext }} / B_{\mathrm{c} 2}(0)=0.44: A_{\mathrm{p}}$ is no longer the saddle, but a local minimum. $B_{\mathrm{p}}$ is at the absolute minimum where the lowest edge of $\tilde{D}_{\mathrm{c}}\left(B^{Z}\right)$ occurs. The saddle points are located in between $A_{\mathrm{p}}$ and $B_{\mathrm{p}}$. Those features are also seen from Fig. 17 in Ref. [9], where the contour plots for the three types of distributions are displayed.

Let us now discuss the physical origins of those three kinds of field profiles. In Fig. 10 we show the current profiles around the vortex core along the path from $V C-A_{\mathrm{p}}$ where each curve corresponds to that in Fig. 9. In GL $\left[B_{\mathrm{ext}} / B_{\mathrm{c} 2}(0)=\right.$ 0.18 ] the current maximum appears relatively near $V C$, and its amplitude quickly decays towards $A_{\mathrm{p}}$. Thus the current curve approaches $A_{\mathrm{p}}$ from above its tangential slope there, implying that the neighboring vortex cores are far apart and the vortex cores are not overlapped. This means that the farthest $B_{\mathrm{p}}$ point from the neighboring vortex cores in a unit cell is a $B_{\text {min }}$ location. In contrast, the DL case shows that the current maximum moves towards $A_{\mathrm{p}}$, with the tangent of the current amplitude being largest among the three profiles. This means that the neighboring vortex cores are densely packed with the vortex cores overlapped, causing $B_{\mathrm{p}}$ not to be the minimum field location in a unit cell. The field profile is quite different from that in GL, making $A_{\mathrm{p}}$ the minimum field location. In the KL limit those features are in between the GL and DL cases.

Figure 11 shows the comparison between the theoretical calculations and the experimental data of the $T$ dependence of $B_{\min }, B_{\mathrm{sad}}$, and $B_{\mathrm{vc}}$ at two $B_{\mathrm{ext}}$ values. The theoretical calculation has been done by varying the $\kappa_{\mathrm{GL}}$ value to best fit those three values near $T / T_{\mathrm{c}}\left(B_{\text {ext }}\right)=1$. It turns out that the

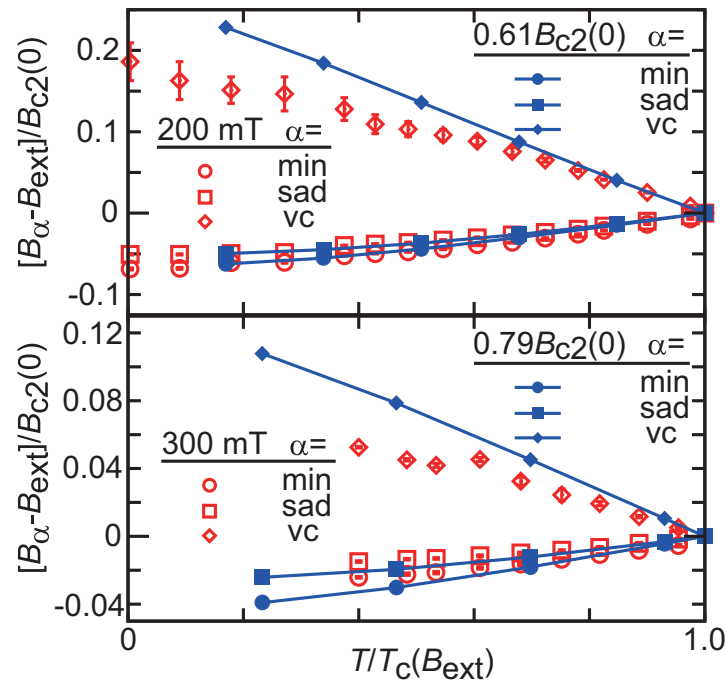

FIG. 11. (Color online) The three characteristic fields $B_{\alpha}(\alpha=$ minimum, saddle, and vortex core) vs the normalized temperature $T / T_{\mathrm{c}}\left(B_{\text {ext }}\right)$ for two values of $B_{\text {ext }}$. The computed values are shown by solid symbols linked by line segments. We have measured $T_{\mathrm{c}}\left(B_{\mathrm{ext}}\right)=$ $5.6 \mathrm{~K}$ and $T_{\mathrm{c}}\left(B_{\text {ext }}\right)=3.95 \mathrm{~K}$ for $B_{\text {ext }}=200$ and $300 \mathrm{mT}$, respectively.

best fitting is achieved for $\kappa_{\mathrm{GL}}=1.8$. This value is two times larger than the nominal value $\kappa_{\mathrm{GL}}=0.89(1)$ of the present sample mentioned before. We notice that the three types of field distributions, namely, the GL, KL and DL cases, are always present irrespective of the choice of $\kappa_{\mathrm{GL}}$. Figure 11 shows the following.

(1) The initial slopes of the three characteristic fields near $T / T_{\mathrm{c}}\left(B_{\text {ext }}\right)=1$ are nicely reproduced for the two $B_{\text {ext }}$ values.

(2) Those nice fittings continue to lower temperatures for $B_{\text {min }}$ and $B_{\text {sad }}$.

(3) In contrast, $B_{\mathrm{vc}}$ starts to deviate towards lower temperatures. While the theoretical curves keep increasing linearly with large slopes, the temperature dependence of the experimental data is much weaker.

According to a previous calculation, $B_{\mathrm{vc}}$ is expected to keep increasing towards zero temperature in the clean limit [23] because of the so-called Kramer-Pesch effect [32]. As already noticed, a previous NMR experiment by Kung [10] on vanadium shows the expected linear temperature dependence of $B_{\mathrm{vc}}$ in a large temperature range towards zero temperature. Clearly, it would be of much interest to perform TF- $\mu$ SR measurements on a very clean vanadium sample to confirm Kung's result and to extend it to very low temperature.

We show the field dependences of the three characteristic fields in Fig. 12. It is seen that the theoretical predictions for $B_{\min }$ and $B_{\text {sad }}$ nicely follow the experimental results, and the qualitative field dependence of $B_{\mathrm{vc}}$ is explained but quantitatively deviates because in those low temperatures the Kramer-Pesch effect is partially suppressed, as mentioned above. Since $B_{\mathrm{vc}}$ reflects the spatial structure around a vortex core, the partially suppressed Kramer-Pesch effect implies that the conical shape structure of $B^{Z}(\mathbf{r})$ at the vortex-core position is rounded relative to theoretical expectation. However, the linear field tail of the distribution at high field, a signature of the conical feature, is nicely observed at low temperature [13]. 


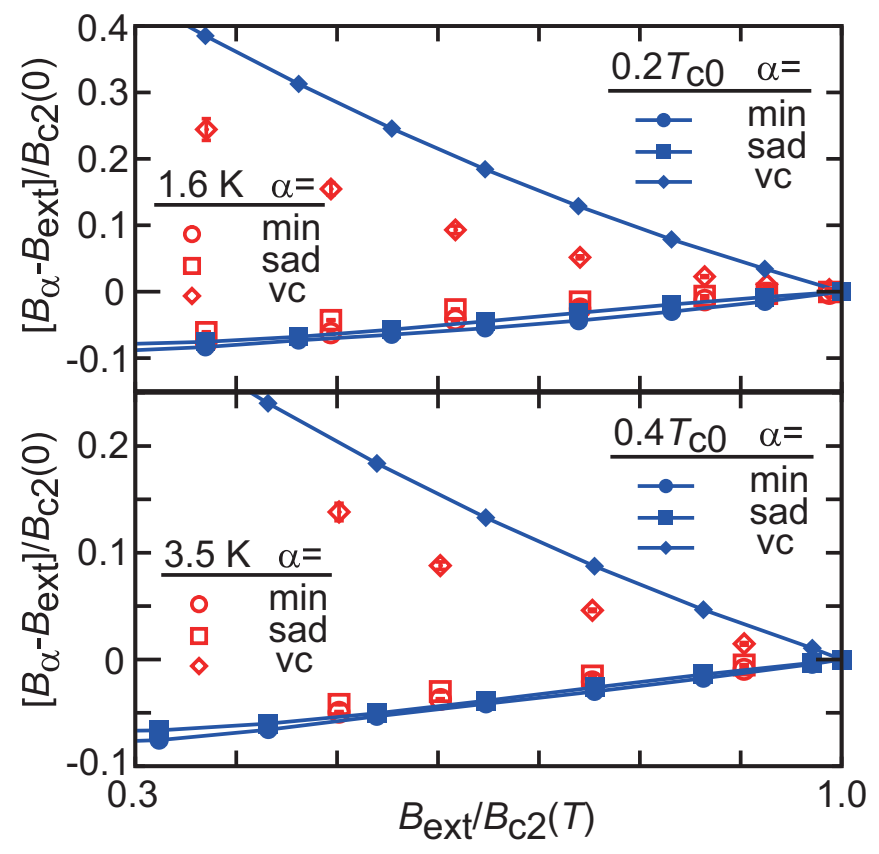

FIG. 12. (Color online) The three characteristic fields $B_{\alpha}(\alpha=$ minimum, saddle, and vortex core) vs the normalized field $B_{\text {ext }} / B_{\mathrm{c} 2}(T)$ at two temperatures. The computed values are shown by the solid symbols linked by line segments. Experimentally, $B_{\mathrm{c} 2}=405$ and $321 \mathrm{mT}$ for $T=1.6$ and $3.5 \mathrm{~K}$, respectively.

\section{VL field map and contour plot}

We have previously established that Delrieu's approximation is reliable for a large part of the mixed phase. The computed $\delta B_{\text {sad,min }}^{\mathrm{n}}$ map displayed in Fig. 13 visualizes

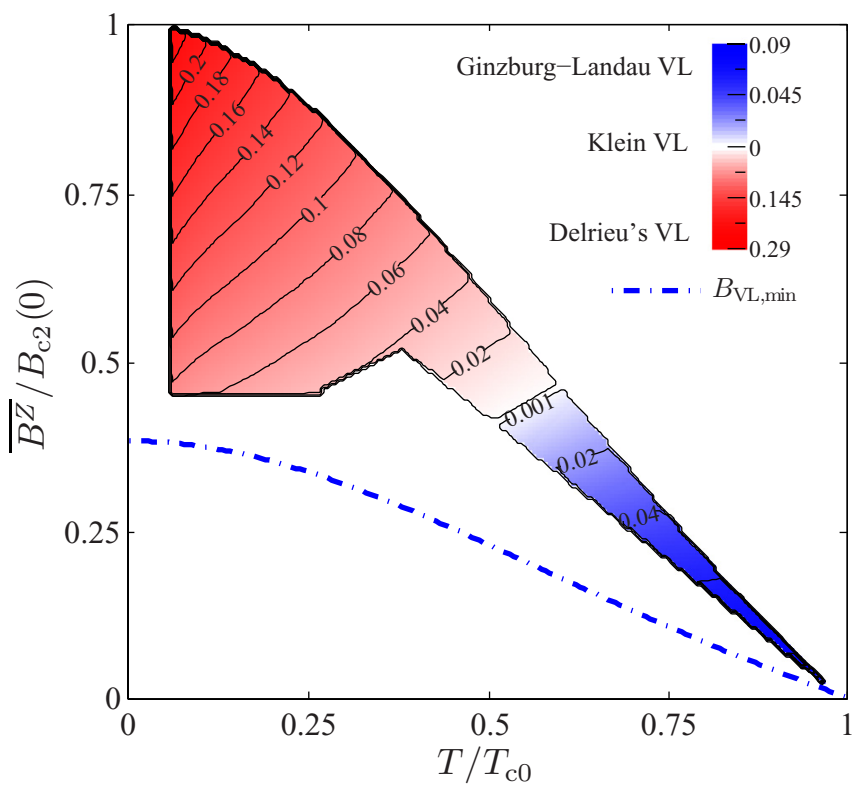

FIG. 13. (Color online) Contour map of $\delta B_{\text {sad,min }}^{\mathrm{n}}$ for niobium with $\mathbf{B}_{\text {ext }} \|\langle 111\rangle$ computed with Delrieu's solution for the form factor. We have restricted the map to the temperature and field regimes properly described by that solution. We specify the regimes consistent with Delrieu, Klein, and Ginzburg and Landau. The dash-dotted line displays $B_{\mathrm{VL}, \min }(T)$. the region of the mixed phase where the approximation is reliable for the analysis of our niobium data. Remarkably, the $\left(T, B_{\text {ext }}\right)$ points close to $B_{\mathrm{c} 2}$ at which $\delta B_{\text {sad,min }}^{\mathrm{n}}=0$ effectively correspond in Fig. 5 to the temperature where the measured $\delta B_{\text {sad,min }}^{\mathrm{n}}$ is minimum. This occurs around $5.5 \mathrm{~K}$. It is because of the Gaussian smearing that $\delta B_{\text {sad,min }}^{\mathrm{n}}$ does not vanish experimentally.

From Delrieu, $\delta B_{\text {sad,min }}^{\mathrm{n}}=0$ at the border between the DL and GL VL region. This border belongs to Klein's regime, which we will discuss now.

In order to examine the diagram obtained experimentally in Fig. 13, we have performed extensive computations to construct the corresponding diagram whose results are displayed in Fig. 14. The overall features in Figs. 13 and 14 coincide, namely, DL occupies the higher-field region while GL is located at lower field. The KL region is in the middle. However, the KL regime is no longer limited to the border between the DL and GL regions: it has an appreciable extension. This is a key result obtained from Eilenberger's solution. Delrieu's approximation is too rough to capture the subtleties in $B^{Z}(\mathbf{r})$ in the KL regime (see Fig. 9). In addition, while in the vicinity of $B_{\mathrm{c} 2}(T)$ the values of $\delta B_{\text {sad,min }}^{\mathrm{n}}$ in Figs. 13 and 14 strikingly correspond for a given field and temperature, outside that regime deviations between the results in the two figures are clearly found: a given $\delta B_{\text {sad,min }}^{\mathrm{n}}$ value in the DL region is only weakly temperature dependent in Fig. 14, in contrast to the results presented in Fig. 13. Before going further in comparing the experimentally deduced and the theoretical diagrams, some comments are in order.

Figure 13 considers $\overline{B^{Z}}$ rather than $B_{\text {ext }}$ because it is this parameter which enters into Delrieu's theory. However, in practice $\overline{B^{Z}} \simeq B_{\text {ext }}$. Because the lower-field border of the measured phase is dependent on the experimental conditions, for the sake of completeness, we have extended the theoretical diagram in Fig. 14 to lower fields and temperatures than in Fig. 13. We stress that while Delrieu's formula does not describe the low-temperature region of the mixed phase as seen

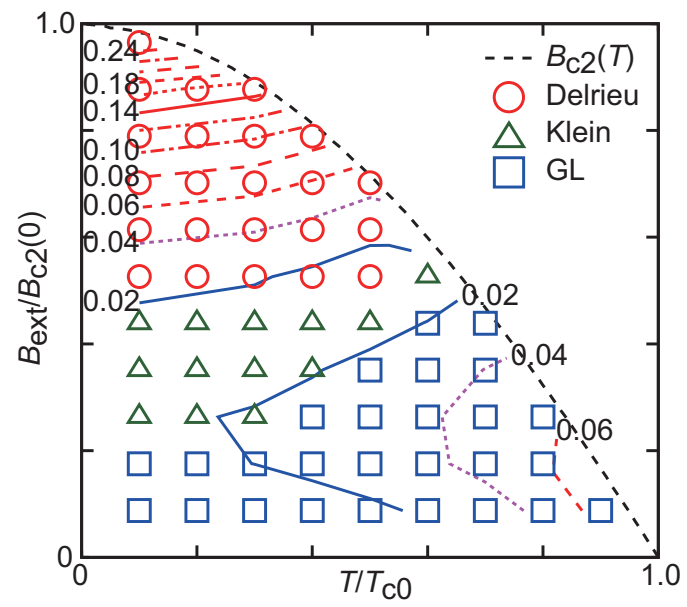

FIG. 14. (Color online) Contour map of $\delta B_{\text {sad,min }}^{\mathrm{n}}$ for niobium with $\mathbf{B}_{\text {ext }} \|\langle 111\rangle$ computed with Eilenberger's equation. In Klein's region two minimal field positions exist; see the inset on the right in Fig. 9. The data in Fig. 6 (Fig. 7) correspond to scanning vertically at low (high) temperature. For the data in Fig. 5 scanning is done parallel to the $B_{\mathrm{c} 2}(T)$ line. 


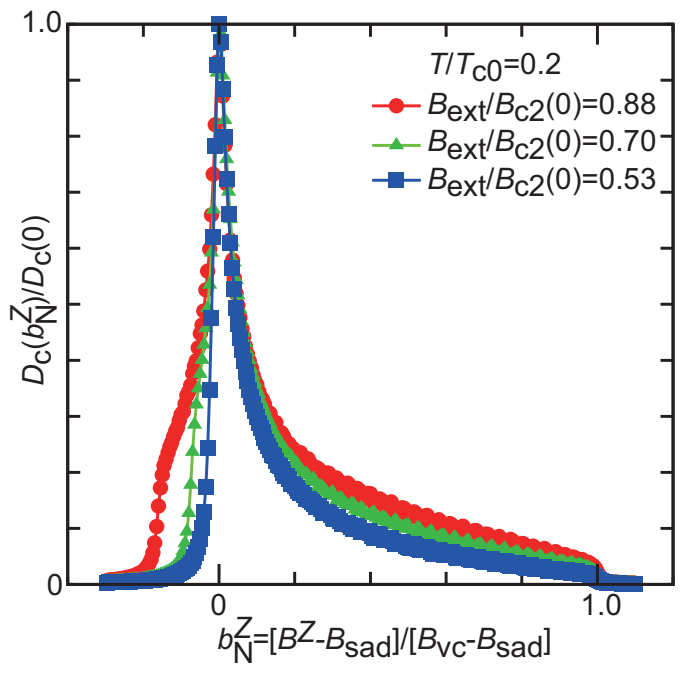

FIG. 15. (Color online) The normalized field distributions $D_{\mathrm{c}}\left(b_{\mathrm{N}}^{Z}\right) / D_{\mathrm{c}}(0)$ for three field values at $T / T_{\mathrm{c} 0}=0.2$ corresponding to the data in the right panel of Fig. 6. Those theoretical results are traversing the map in Fig. 14 vertically.

in Fig. 13, the numerical solution of Eilenberger's equation is expected to provide a proper description.

The data set shown in Fig. 6 corresponds to scanning the field at a fixed low temperature in Fig. 14. As this map implies, $\delta B_{\text {sad,min }}^{\mathrm{n}}$ increases as $B_{\text {ext }}$ increases. In fact, as seen from Fig. 15, where we show the computed normalized field distributions $D_{\mathrm{c}}\left(b_{\mathrm{N}}^{Z}\right) / D_{\mathrm{c}}(0)$ under a fixed temperature $\left(T / T_{\mathrm{c} 0}=0.2\right)$ for three field values, as $B_{\mathrm{ext}}$ increases, $B_{\min }$ moves to the lower-field side, i.e., to the left of the field scale. This explains the fact that in Fig. $6 B_{\min }$ increases in absolute value as $B_{\text {ext }}$ increases.

As for Fig. 5, where the scanning path is taken parallel to the $B_{\mathrm{c} 2}(T)$ line, it is seen from Fig. 14 that $\delta B_{\text {sad,min }}^{\mathrm{n}}$ decreases first and then increases towards lower temperatures, coinciding with the data in Fig. 5.

Finally, as for GL, Fig. 7 shows that the two distributions are hardly distinguishable because the two distributions are both inside the GL region in Fig. 14, where the GL distribution is universal and scaled.

Those various scanning data throughout the $B_{\text {ext }}-T$ plane demonstrate precise correspondence between Eilenberger's theory and experiment, supporting the existence of the three distinct characteristic field distributions, i.e., the GL, KL, and DL distributions.

\section{SUMMARY OF THE RESULTS OBTAINED IN THIS STUDY AND POSSIBLE IMPROVEMENTS OF THE ANALYSIS AND DATA RECORDING}

In summary, combining TF- $\mu$ SR measurements analyzed with Delrieu's analytical solution for the form factor, supplemented with conventional phenomenological formulas for the physical parameters, and the numerical solutions of the quasiclassical Eilenberger equation to get $B^{Z}(\mathbf{r})$, we have established that the VL of niobium with $\mathbf{B}_{\text {ext }}$ applied along a three-fold axis is characterized by three successive regions as the sample is cooled down from $T_{\mathrm{c} 0}$. Hence, our work supports the predictions of Abrikosov, Klein, and Delrieu, respectively.

The experimental data exhibit three regions in the mixed phase which are explained by Eilenberger's theory with cylindrical Fermi surface. However, quantitative deviations in the field and temperatures dependence of $B_{\mathrm{vc}}$ from theory are evident. Surprisingly, a $\kappa_{\mathrm{GL}}$ parameter twice as large as the measured value has to be assumed. We know of two sources for possible explanations of the discrepancies.

(1) Our numerical solution of Eilenberger's equation does not take into account the Fermi velocity anisotropy and gap anisotropy known to exist, as seen in the $B_{\mathrm{c} 2}$ anisotropy $[33,34]$. In particular the Fermi velocity anisotropy generally increases the $B_{\mathrm{c} 2}$ value, thus causing the estimate of $\kappa_{\mathrm{GL}}$ to change.

(2) We regard $\kappa_{\mathrm{GL}}$ as an effective parameter because the theory assumes the clean limit. Although our sample is extremely clean [13], it is known that defects and impurities act to increase $\kappa_{\mathrm{GL}}$ from the nominal value.

We have to deal with three sources of field distributions at the muon site: the nuclear ${ }^{93} \mathrm{Nb}$ magnetic moments, the VL itself, and the effect of the VL disorder. To a good approximation, the component field distribution from the nuclear moments in a TF- $\mu$ SR experiment is Gaussian [12]. We have just discussed how the description of the distribution from the VL itself can be improved. It is known that modeling the effect of the VL disorder with a Gaussian field function as done in this paper is a rough approximation. A close look at Fig. 2 of Ref. [13] shows it definitively, particularly in the vicinity of the low-field tail. In fact, the translational correlations of the vortex cores are neglected in the Gaussian approximation [35]. To progress we need to recognize that the $\mathrm{VL}$ is not a two-dimensional lattice but a three-dimensional lattice; that is, we are dealing with the flux-line lattice (FLL). As for any lattice, disorder has to be considered. In the FLL case we need to remember that the collective behavior matters [36]. In the absence of dislocations, if disorder is not too strong the FLL is periodic, as clearly demonstrated by SANS measurements, but the FLL translational order decays only algebraically rather than exponentially $[37,38]$, as expected theoretically [39]. In fact, a so-called Bragg glass state is expected [40] and observed [37,38]. However, we stress that it was found for samples with appreciable disorder. It is still a challenge to observe it for a clean sample such as ours. A numerical method to account for the Bragg glass state has been devised for the analysis of SANS measurements [38]. This has yet to be done for the $\mu$ SR counterpart.

Neither Delrieu's analytical solution nor the numerical solution of Eilenberger's equation describes the measured distributions below $0.6 \mathrm{~K}$ [13]. A proper account of the vortex-lattice residual disorder may round up the predicted sharp conical field shape at the VL vortex cores and explain the measurements below $0.6 \mathrm{~K}$.

Up to now we have discussed possible improvements of the data analysis. But the experimental conditions themselves could also be optimized. All the TF- $\mu$ SR asymmetry time spectra have been recorded on a single crystal disk with $\mathbf{B}_{\text {ext }}$ parallel to the disk axis; see the pictogram in Fig. 3. In this geometry inhomogeneities due to the demagnetization field near the sample boundaries may have to be considered. An 
improved experimental setup would require us to apply $\mathbf{B}_{\text {ext }}$ perpendicular to the disk axis. However, for the necessary high $B_{\text {ext }}$ values this is not possible since the positive muon is a charge particle and as such would be deflected from its trajectory according to the Lorentz force. Hence, $\mathbf{B}_{\text {ext }}$ should be kept in the direction we have chosen. Therefore, to improve on the experimental conditions, an elliptical single sample would have to be used.

The form factors of a vortex lattice can be studied with the SANS technique. It is well known that the exact solution of GL theory gives some $K_{m, n}$ form factors of opposite sign relative to those predicted by analytical approximations of GL theory or the London model; see Ref. [22] for a discussion. Regarding Delrieu's solution, the signs of the form factors are the same as is in Abrikosov's solution and are given by the factor $(-1)^{m h}$ [20]. Only the magnitude of the form factors varies with the values of parameters $\tilde{b}$ and $\tilde{c}$. The signs of the form factors at high order from the Eilenberger solution still have to be evaluated. This requires getting the solution accurate enough to extract those higher-order harmonics because they become extremely small numbers.

\section{CONCLUSIONS AND PERSPECTIVES}

In conclusion, combining the TF- $\mu$ SR experimental technique with Delrieu's analytical solution and numerical solutions of the quasiclassical Eilenberger equation, we have observed the three theoretically expected regions in the mixed phase of niobium with $\mathbf{B}_{\text {ext }}$ applied along a three-fold axis. We do not know of any previous experimental observation of the three regions. Our results should apply to any clean $s$-wave superconductor with a triangular vortex lattice.

The experimental data have been recorded at high statistics, and the analysis has been done with advanced methods. Possible improvements of the data analysis and experimental conditions have been pointed out. We hope that our work will motivate people to analyze $\mathrm{TF}-\mu \mathrm{SR}$ asymmetry time spectra for other $s$-wave superconductors with the framework presented here. An obvious candidate is vanadium, for which the sample should be in the extremely clean limit.

\section{ACKNOWLEDGMENTS}

We thank P. Dalmas de Réotier for a careful reading of the manuscript. The $\mu \mathrm{SR}$ measurements were performed at Swiss Muon Source $(\mathrm{S} \mu \mathrm{S})$, Paul Scherrer Institute (PSI), Villigen, Switzerland. We acknowledge partial support from NCCR MaNEP, sponsored by the Swiss National Science Foundation. K.M. was supported by JSPS (Grants No. 21340315 and No. 9134919315) and "Topological Quantum Phenomena" (Grant No. 2510371615) KAKENHI on Innovation Areas from MEXT.
[1] M. Tinkham, Introduction to Superconductivity (McGraw-Hill, New York, 1996).

[2] J. B. Ketterson and S. N. Song, Superconductivity (Cambridge University Press, Cambridge, 1999).

[3] A. A. Abrikosov, Sov. Phys. JETP 5, 1174 (1957).

[4] L. P. Gor'kov, Sov. Phys. JETP 9, 1364 (1959).

[5] J. E. Sonier, J. H. Brewer, and R. F. Kiefl, Rev. Mod. Phys. 72, 769 (2000).

[6] J. E. Sonier, Rep. Prog. Phys. 70, 1717 (2007).

[7] J. M. Delrieu, J. Low Temp. Phys. 6, 197 (1972).

[8] G. Eilenberger, Z. Phys. 214, 195 (1968).

[9] U. Klein, J. Low Temp. Phys. 69, 1 (1987).

[10] A. Kung, Phys. Rev. Lett. 25, 1006 (1970).

[11] P. Dalmas de Réotier and A. Yaouanc, J. Phys.: Condens. Matter 9, 9113 (1997).

[12] A. Yaouanc and P. Dalmas de Réotier, Muon Spin Rotation, Relaxation, and Resonance: Applications to Condensed Matter, International Series of Monographs on Physics Vol. 147 (Oxford University Press, Oxford, 2011).

[13] A. Maisuradze, A. Yaouanc, R. Khasanov, A. Amato, C. Baines, D. Herlach, R. Henes, P. Keppler, and H. Keller, Phys. Rev. B 88, 140509 (2013).

[14] J. Schelten, H. Ullmaier, and W. Schmatz, Phys. Status Solidi B 48, 619 (1971).

[15] R. Kahn and G. Parette, Solid State Commun. 13, 1839 (1973).

[16] E. M. Forgan, S. J. Levett, P. G. Kealey, R. Cubitt, C. D. Dewhurst, and D. Fort, Phys. Rev. Lett. 88, 167003 (2002).

[17] S. Mühlbauer, C. Pfleiderer, P. Böni, M. Laver, E. M. Forgan, D. Fort, U. Keiderling, and G. Behr, Phys. Rev. Lett. 102, 136408 (2009).
[18] D. Herlach, G. Majer, J. Major, J. Rosenkranz, M. Schmolz, W. Schwarz, A. Seeger, W. Templ, E. H. Brandt, U. Essmann, K. Fürderer, and M. Gladisch, Hyperfine Interact. 63, 41 (1991).

[19] N. R. Werthamer, in Superconductivity, edited by R. D. Parks (Marcel Dekker, New York, 1969), Vol. 1, pp. 321-370.

[20] A. Maisuradze and A. Yaouanc, Phys. Rev. B 87, 134508 (2013).

[21] H. M. Adachi, M. Ishikawa, T. Hirano, M. Ichioka, and K. Machida, J. Phys. Soc. Jpn. 80, 113702 (2011).

[22] E. H. Brandt, Phys. Rev. Lett. 78, 2208 (1997).

[23] P. Miranović and K. Machida, Phys. Rev. B 67, 092506 (2003).

[24] M. Laver, E. M. Forgan, S. P. Brown, D. Charalambous, D. Fort, C. Bowell, S. Ramos, R. J. Lycett, D. K. Christen, J. Kohlbrecher, C. D. Dewhurst, and R. Cubitt, Phys. Rev. Lett. 96, 167002 (2006).

[25] E. H. Brandt, Phys. Status Solidi B 65, 469 (1974).

[26] A. Maisuradze, R. Khasanov, A. Shengelaya, and H. Keller, J. Phys.: Condens. Matter 21, 075701 (2009).

[27] A. Yaouanc, P. Dalmas de Réotier, and E. H. Brandt, Phys. Rev. B 55, 11107 (1997).

[28] M. Ichioka, N. Hayashi, and K. Machida, Phys. Rev. B 55, 6565 (1997).

[29] M. Ichioka, A. Hasegawa, and K. Machida, Phys. Rev. B 59, 8902 (1999).

[30] M. Ichioka, A. Hasegawa, and K. Machida, Phys. Rev. B 59, 184 (1999).

[31] N. Nakai, P. Miranović, M. Ichioka, and K. Machida, Phys. Rev. B 73, 172501 (2006).

[32] W. Pesch and L. Kramer, J. Low Temp. Phys. 15, 367 (1974). 
[33] S. J. Williamson and L. E. Valby, Phys. Rev. Lett. 24, 1061 (1970).

[34] S. J. Williamson, Phys. Rev. B 2, 3545 (1970).

[35] A. Yaouanc, A. Maisuradze, and P. Dalmas de Réotier, Phys. Rev. B 87, 134405 (2013).

[36] M. V. Feigel'man, V. B. Geshkenbein, A. I. Larkin, and V. M. Vinokur, Phys. Rev. Lett. 63, 2303 (1989).
[37] T. Klein, I. Joumard, S. Blanchard, J. Marcus, R. Cubitt, T. Giamarchi, and P. L. Doussal, Nature (London) 413, 404 (2001).

[38] M. Laver, E. M. Forgan, A. B. Abrahamsen, C. Bowell, T. Geue, and R. Cubitt, Phys. Rev. Lett. 100, 107001 (2008).

[39] T. Nattermann, Phys. Rev. Lett. 64, 2454 (1990).

[40] T. Giamarchi and P. Le Doussal, Phys. Rev. B 52, 1242 (1995). 